\title{
EGFR NP_005219.2:p.V834del
}

National Cancer Institute

\section{Source}

National Cancer Institute. EGFR NP 005219.2:p.V834del. NCI Thesaurus. Code C98589.

A deletion of the valine at position 834 from the epidermal growth factor receptor

protein. 